Mythe et histoire

\title{
Mythe et histoire dans la Sophonisbe de J. Marston
}

Jacqueline Chauchaix

\section{Q OpenEdition \\ Journals}

Édition électronique

URL : http://journals.openedition.org/shakespeare/499

DOI : 10.4000/shakespeare.499

ISSN : 2271-6424

\section{Éditeur}

Société Française Shakespeare

Édition imprimée

Date de publication : 1 novembre 1984

Pagination : 127-146

Référence électronique

Jacqueline Chauchaix, "Mythe et histoire dans la Sophonisbe de J. Marston », Actes des congrès de la Société française Shakespeare [En ligne], 5| 1984, mis en ligne le 01 janvier 2007, consulté le 01 mai 2019. URL : http://journals.openedition.org/shakespeare/499 ; DOI : 10.4000/shakespeare.499 
SOCIETE FRANÇAISE SHAKESPEARE

Actes du Congrès 1983

\section{MYTHE ET HISTOIRE}

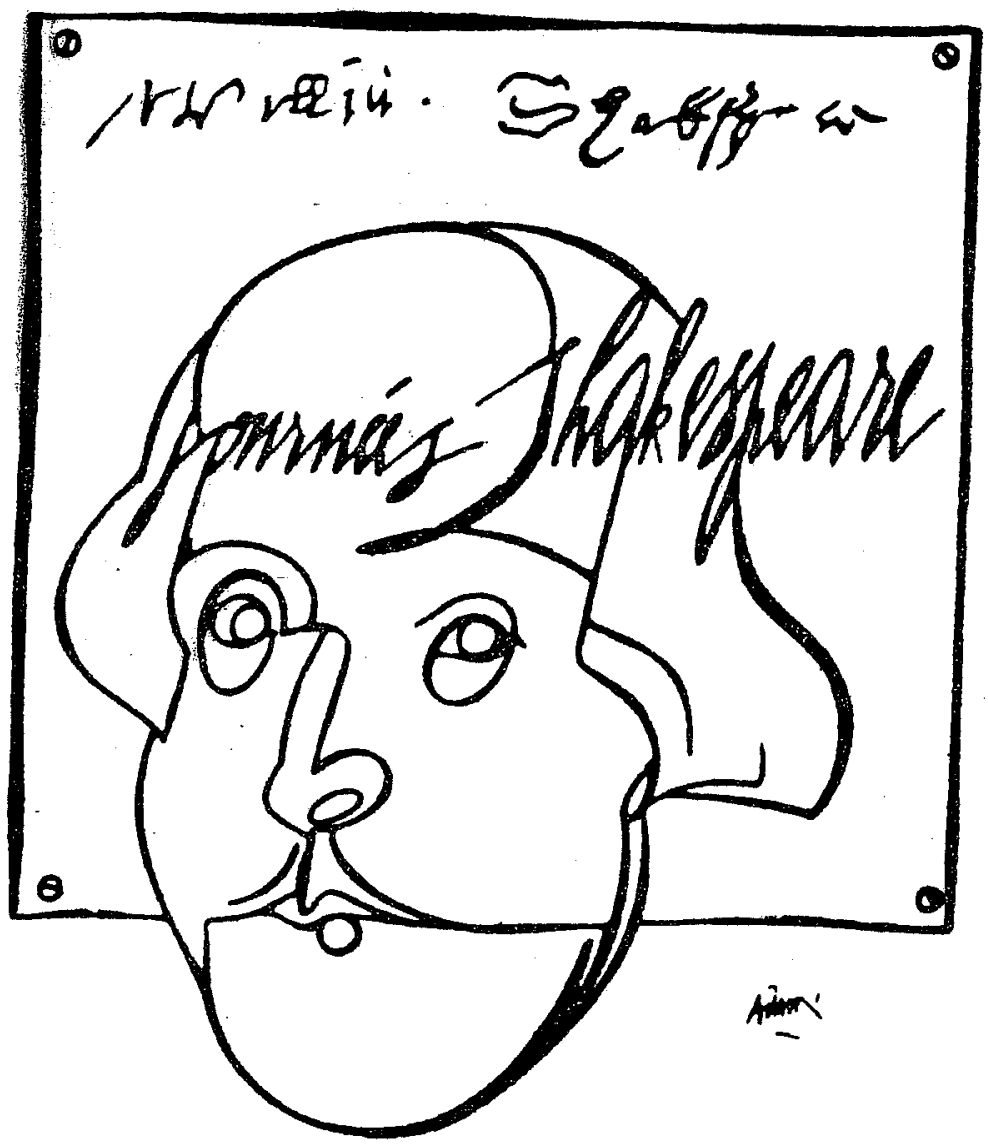

$$
\begin{array}{r}
\text { DiReCteur DE LA' PUblicatic } \\
\text { M.T. Jones-Davie }
\end{array}
$$

Publié avec le concours du Centre National de la Recherche Scientifiq

$$
\text { JEAN TOUZOT Libraire - Editeur }
$$

38, rue Saint-Sulpice 75278 PARIS CEDEX 06199 


\section{HISTOIRE ET MYTHE DANS LA SOPHONISBE DE J. MARSTON}

La Sophonisbe de J. Marston et les récits historiques qui en sont la source n'étant pas forcément les livres de chevet de chacun, je rappellerai que Sophonisbe est une princesse carthaginoise qui se suicida par patriotisme vers la fin de la deuxième guerre punique. Divers historiens antiques ont raconté, plus ou moins longuement, cet épisode : Polybe, Tite-Live, Diodore de Sicile, Appien et Dion Cassius, parmi d'autres. De nombreuses versions littéraires, c'est-à-dire des relations qui ne sont pas sous-tendues par une revendication de véracité, ont été données. Des dizaines d'hommes de lettres, des dramaturges essentiellement, italiens, français, anglais et allemands, ont repris les données historiques fondamentales de cet épisode tout en les pliant à la mode du moment ${ }^{1}$. A travers ces variations, on peut repérer des invariants, par exemple la présence constante de quatre personnages (Sophonisbe, Scipion, Massinisse et Syphax) et de certains moments essentiels qui sont les principaux points d'appui du récit (l'arrivée des Romains en Afrique, la défaite des Carthaginois, le mariage de Sophonisbe et de Massinisse, et le suicide de Sophonisbe). Les nombreuses versions littéraires qui ont été données de cet épisode de l'histoire antique semblent indiquer qu'il a eu une forte emprise sur l'imagination, qu'il a été une «puissance d'investissement de la sensibilité» $^{2}$. Si l'aptitude à renâitre, la plasticité et l'efficacité émotionnelle sont des critères du mythe littéraire, la question peut légitimement être posée de savoir si l'histoire de Sophonisbe s'est constituée en my the:

Or force nous est de constater que le personnage de Sophonisbe n'est pas devenu une référence pour la méditation. Son nom n'est pas devenu un mot de passe ; un mythe ne semble pas avoir cristallisé autour de lui. Si l'on admet que tout objet peut devenir la proie de la parole mythique ${ }^{3}$, sans doute faut-il conclure que ce per- 
sonnage et son histoire n'ont pas trouvé d'auteur assez puissant pour leur donner une dimension mythique. Si l'on pense, inversement, que le mythe est affaire d'objet et non de façon, sans doute faut-il conclure que ce personnage ne peut pas se hausser jusqu'au statut de héros mythique ${ }^{4}$. Peut-être, en effet, le suicide patriotique de Sophonisbe ne suffit-il pas pour que le personnage échappe à la banalité de la simple chronique. Les diverses jeunes filles de l'Acropole, par exemple les filles d'Erechthée, qui sacrifièrent leur vie par dévotion à leur patrie, restent des figures mineures de la mythologie antique. Le courage qui pousse Antigone à se soulever contre un interdit est peutêtre, aujourd'hui en tout cas, plus prisé que la piété patriotique. Et pourtant, dans le personnage de Jeanne d'Arc, les hommes déchiffrent un sens qui leur importe aujourd'hui encore. $\mathrm{Y}$ a-t-il dans le personnage de Sophonisbe une contradiction inhérente qui l'empêche a priori d'accéder à un destin mythique? Par son énergie, sa force d'âme, Sophonisbe, la Carthaginoise 5 , est, en effet, un abrégé des vertus romaines.

La Sophonisbe de J. Marston, publiée en 1606, se définit, de même que les Sophonisbe qui l'ont précédée et celles qui lui ont succédé, par un double rapport d'opposition à l'histoire et au mythe. C'est la tension qui existe entre ces deux pôles, dans la pièce du dramaturge anglais, que je vais tenter d'examiner.

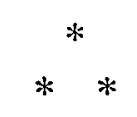

Marston a lu les historiens antiques 6 . Divers emprunts irréfutables indiquent qu'il a lu Tite-Live et Appien ; peut-être a-t-il aussi. lu Diodore de Sicile et Dion. Cassius. Il emprunte à Tite-Live et à Appien tout un arrière-plan de noms de personnages et de lieux, un canevas événementiel, quelques notations topographiques et quelques détails de couleur locale et historique, qui servent d'ancrage référentiel.

Et pourtant, le texte ne se prête pas au juge- 
ment de vérité historique. On y trouve, non pas une fidélité archéologique, semblable à celle qu'a recherchée Flaubert dans Salammbô, mais une antiquité de pacotille ${ }^{7}$. Le message narratif et le message idéologique ont subi des transformations qui semblent indiquer que l'histoire a été infléchie en direction du mythe, tant du point de vue du contenu que de l'organisation du contenu.

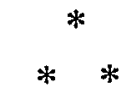

De manière générale, Marston fait subir à son sujet une mutation par laquelle il en rajeunit le sens. Divers thèmes de morale politique et de morale privée, déjà présents dans les récits historiques, ont été amplifiés, précisés ou modulés par l'apport de courants de pensée (chrétien et machiavélien, par exemple) et de modes de sensibilité (pastoral et baroque entre autres) caractéristiques de l'époque de Marston. Le thème de la fidélité à autrui et à soi-même, en particulier, s'est chargé de nouvelles composantes historiques : l'apologie de la constan$c e$, le retour insistant de ce thème sont liés au contexte politique, social, moral, religieux et scientifique, dans lequel la pièce a été produite. Au tournant du siècle, les hommes font l'expérience de l'inconstance : rien n'est stable. Bref, Marston ancre sa fiction dans l'actualité et transfère à son époque le sujet qu'il a choisi dans des temps anciens.

Il fait plus, d'ailleurs, que raccorder deux temporalités différentes. On trouve dans la pièce, en effet, une certaine façon de penser le temps qui relève, en partie tout au moins, de la pensée mythique. Certes, l'action est ancrée dans l'histoire par un calendrier externe (il $\mathrm{y}$ a un avant et un après) et un calendrier interne (une chronologie interne régit les événements relatés). Mais le récit glisse à plusieurs reprises vers l'atemporalité parce que Marston, par des procédés divers, brouille et estompe les limites temporelles et les repères chronologiques internes. Quittant l'anecdote, il emprunte de-ci de-là 
à des passés différents. La liste des noms de personnages, par exemple, est un habit d'Arlequin : certains noms ont appartenu à des personnages historiques du $\mathrm{I}$ er siècle avant notre ère, d'autres ont été empruntés à des personnages du Ile ou du IIle siècle avant Jésus-Christ ; un nom est calqué, peu s'en faut, sur celui d'un personnage de l'histoire moderne ${ }^{8}$. Plus généralement, par un mouvement d'abstraction ${ }^{9}$, Marston ote aux personnages leur couleur antique. Il atténue la marque du temps. L'action progresse parfois par juxtaposition de tableaux statiques dont certains pourraient être intervertis sans inconvénient ${ }^{10}$. Il arrive aussi que soit rapporté au présent (historique) un événement donné comme ayant eu lieu dans le passé (II, 3, v. 89-90, p. 269), alors qu'il est montré sur scène quelques minutes plus tard (III, 2, v. 35-38, p. 283) ${ }^{11}$. L'action amoureuse est remarquable par son immobilité ou, disons, sa structure circulaire. Ni Massinisse, ni Syphax ne parvient à assouvir le désir dont Sophonisbe est l'objet : rien ne se passe d'un bout à l'autre de la pièce ${ }^{12}$. . D'entrée de jeu, le Prologue fait tenir passé et futur dans le présent, englobant ainsi la totalité du temps : d'une part, en effet, le présent qu'il utilise rend contemporains personnages et spectateurs en insérant, dans le présent du spectateur (Prologue, v. 10-11, p. 237), une suite d'événements passés (v. 2-9, p. 237 et v. 12-13, p. 238) ; d'autre part, il raconte au présent ce qui va avoir lieu (v. 14-18, p. 238). La durée est momentanément suspendue. Le récit semble s'affranchir de la linéarité historique 13 .

L'intention poétique avouée par Marston dans l'Epître au lecteur situe la pièce dans un double rapport au faux et au vrai qui est lui aussi caractéristique de la pensée mythique : «Know that I have not laboured in this poem to tie myself to relate anything as an historian, but to enlarge everything as a poet» ${ }^{14}$. Marston se dit «poète» parce qu'il invente une fiction qui exprime un sens caché. Il s'inscrit dans le courant humaniste platonicien qui, en mettant l'accent sur l'origine divine de l'inspiration poétique, attribue au poète, créateur de my- 
thes, le pouvoir de révéler des vérités ${ }^{15}$. Au-delà du monde sensible des personnages et de leurs actions, en effet, au-delà du motif theâtral apparent, se manisfeste un autre motif - «an under-pattern», «a pattern behind the pattern», a écrit T. S. Eliot ${ }^{16}:$ : les personnages conforment leur conduite aux lois d'une réalité invisible, mais qui pourtant est là, d'un monde de vérités immuables. Comme le récit mythique, l'histoire fausse que raconte Marston est douée d'un sérieux tout particulier ( «Noble true story», Epilogue, v. 7, p. 315). Elle se définit par sa haute importance parce que, sous le voile de la fiction, elle invite à déchiffrer un sens.

L'intrusion de la fiction et du mythe dans le récit est signalée, en surface, par des références explicites à des êtres surnaturels. Ces références, au nombre d'une centaine, sont réparties de manière diffuse dans la pièce. Il est à noter qu'aucune divinité africaine ou punique n'est jamais désignée par les Africains ou les Carthaginois. Or au IIle siècle avant Jésus-Christ, les divinités puniques n'étaient pas encore honorées sous des vocables latins. Leur nom fut romanisé un siècle plus tard, sous l'occupation romaine ${ }^{17}$. Cet anachronisme ne surprend pas vu que Marston ne se préoccupait guère d'exactitude historique. Les références à la mythologie latine sont de loin les plus nombreuses, mais on note aussi, en dépit du contexte païen, quelques références à la mythologie biblique. Seule semble significative la fréquence des références à Jupiter, qui est nommé une vingtaine de fois et derrière lequel se dessine la figure du Dieu des Chrétiens. L'expression vague «le Ciel», utilisée à plusieurs reprises, permet à Marston de fondre les croyances anciennes et modernes. Dans l'économie générale de l'action, les dieux ne sont néanmoins que des participants circonstanciels. Les personnages les prennent à témoin, les implorent, les maudissent ou lës défient, soit dans la plénitude de leur foi, soit avec une arrière-pensée politique, soit à des fins strictement égoïstes. Mais la nécessité qui pèse sur l'action est d'ordre naturel. Les dieux, au balcon, si l'on peut dire, sont des spectateurs de l'action. Souvent, ils regar- 
dent en surplomb une scène vide de présence divine, où s'agitent des humains ${ }^{18}$. Marston habille donc l'histoire antique d'un vernis mythologique, mais il fait de la mythologie antique un usage relativement discret, beaucoup plus discret que dans ses poèmes où l'appareil mythologique était particulièrement pesant et pédant. Dans la pièce, les références, loin d'être l'indicateur d'un jeu d'érudit auquel se prêteraient récepteurs et émetteurs d'un message codé, appartiennent à un fonds culturel commun à Marston et à son époque.

Quant aux figures mythiques qui informent le texte de manière implicite, il ne semble pas que l'oeuvre dans son ensemble, ni aucun des personnages, soit sous le signe de l'influence continue' d'un même mythe. Marston juxtapose des résidus mythiques d'origine diverse. Afin d'illustrer la manière dont il démantèle et assemble des mythes divers, je m'en tiendrai à la figure de la sorcière Erich tho, personnage de pure fiction, noyau mythique par excellence.

Erichtho n'apparaît nulle part dans le récit des guerres puniques. Elle apparaît, de manière fugace, dans les Héroides d'Ovide et , plus longuement, dans La Pharsale de Lucain ${ }^{19}$. Dans la pièce, elle est incontestablement apparentée à son homologue de La Pharsale : elle est un personnage d'épouvante, par son allure physique et ses agissements cruels. Mais Marston ne se contente pas de traduire ni de plagier Lucain ${ }^{20}$. Il emprunte divers traits à d'autres figures mythiques. Ainsi, à l'inverse de son homologue de La Pharsale, mais comme Médée, Erichtho allie magie et érotisme : elle convoite la couche d'un roi depuis plus de soixante ans. C'est en cela surtout que Marston innove par rapport à Lucain. A l'inverse de Médée pourtant, elle ne peut pas mettre sa technique au. service de sa passion, puisque la magie est tenue pour inopérante dans le domaine amoureux ${ }^{21}$. Il lui faut avoir recours à une ruse afin d'attirer à elle Syphax. Elle rappelle aussi ces démons femelles des anciens Grecs, les Lamies (c'est-à-dire les gloutonnes, de «laimos», le gosier) qui revêtaient la forme de la beauté afin de s'unir 
aux jeunes hommes dont elles suçaient le sang alors qu'ils dormaient. Erichtho, en effet, parvient à tromper Syphax en lui apparaissant voilée sous les traits de Sophonisbe. Après s'être accouplée à lui, elle l'informe qu'elle a bu son sang, sa jeunesse et sa vie, puis elle se dérobe (V. 1, v. 21-22, p. 298). Le thème de la disparition de l'être aimé, ou présumé tel, semble a priori en tout cas, lié à celui de la rupture de l'interdit visuel. Il ne peut manquer de rappeler le mythe d'Orphée et d'Eurydice et celui d'Eros et de Psyché. Syphax ose poser le regard sur Erichtho, enfreignant ainsi la prescription qu'il a reçue d'elle. Son bonheur s'écroule alors, comme celui d'Orphée lorsqu'il voit ce qu'il ne devait pas voir. Comme Eurydice, Erich tho ne donne finalement à saisir que du vide. Comme Eros, dans le mythe que raconte Apulée ${ }^{22}$, Erichtho qui, par certains aspects tout au moins, échappe à l'humaine condition, s'accouple à un personnage humain qui ne doit pas chercher à le voir. Syphax, comme Psyché, est abandonné pour avoir transgressé l'interdit. Certaines données sont néanmoins totalement transformées : la sorcière hideuse est l'antithèse d'Eros, «le monstre le plus aimable et le plus doux» 23 . La séparation d'Eros et de Psyché est temporaire : après une longue quête, épreuve initiatique, Psyché se trouve établie dans un statut supérieur ; elle devient déesse. En revanche, après avoir perdu l'objet présumé de son amour, Syphax est établi dans un statut inférieur ; il tombe en enfer (V. 1, v. 23, p. 298 et v. 41, p. 299). L'épisode d'Erichtho n'est pas, comme le my the d'Eros et de Psyché, ouvert à une interprétation platonicienne : Erichtho, être infernal qui entre au corps de Syphax, est une inversion d'Eros, ce dieu auquel l'âme s'unit après une longue errance. La figure de la magicienne antique a été également modifiée par des emprunts à l'épóque de Marston et à diverses croyances de cette époque. Ainsi, telles les sorcières terrestres que l'on brûlait alors, Erichtho est une vieille femme hideuse. Elle est, selon certaines croyances que propageait cette époque, incapable de faire naître l'amour par des charmes ou des philtres. $\mathrm{Ou}$ encore, telle un démon invulnérable, elle peut dispa- 
raître à volonté (V, 1, indic. scénique, p. 298 ${ }^{24}$. Elle est une puissance infernale qui a trouvé en Syphax une proie facile parce que, selon une longue tradition chrétienne, il existe entre les démons et les mélancoliques, une sorte d'affinité élective ${ }^{25}$. Plus précisément, et en dépit du contexte païen, elle est un avatar du diable. Elle empêche Syphax d'y voir clair, puis l'entraîne en enfer, dans une chute sans fin ${ }^{26}$. Comme l'esprit démoniaque qui apparaît au Docteur Faust sous la forme d'Hélène, et qui arrache à Faust son âme, Erichtho assure la damnation de Syphax après avoir aveuglé sa conscience. Ce n'est pas, en fait, la connaissance interdite et dérobée mais l'hybris et l'ensommeillement de l'âme qui sont condamnés. Ce n'est pas, dans une perspective néo-platonicienne, la docte ignorance, l'amour aveugle qui sont prônés, mais au contraire la lumière et la lucidité. L'épisode illustre en contre-exemple la leçon des paraboles évangéliques : il faut garder les yeux ouverts et sa lampe allumée. Enfin, certains thèmes investis dans le personnage d'Erichtho tel que l'a conçu Marston sont communs à des mythologies et des traditions diverses - ainsi, par exemple le thème de l'illusion associé à celui de l'amour. Peut-être, après tout, l'apparition d'Erich tho n'est-elle qu'un fantasme, c'està-dire un fantôme de l'imagination de Syphax ? Syphax s'exclame en effet contre l'illusion dont il est victime, contre l'échauffement de ses sens et de son imagination (V, 1, v. 5, p. 297). Comme Héra s'était cachée derrière une nue, à laquelle Ixion, dans son ivresse, s'était uni, Erichtho déguisée en Sophonisbe n'est peut-être qu'une créature illusoire, qui a comblé Syphax par une étreinte imaginaire, un viol fantasmatique. Divers théoriciens de l'époque de Marston tenaient, en effet, l'apparition de succubes pour totalement illusoire ${ }^{27}$. Peut-être aussi le thème de la mort physique lié à celui de l'amour s'inscrit-il dans le schéma de la représentation symbolique de l'acte sexuel. Erichtho permet à Syphax une victoire sur la pesanteur («Whither is Syphax heaved ?» dit Syphax de lui-même, IV, 1, v. 182, p. 294). Il rêve d'un essor qui lui permette de ravir aux dieux leurs privilèges («We'll 
joy such delight,/ That gods shall not admire, but even spite !», IV, 1, v. 218-219, p. 296). Vient ensuite le moment de cécité, la «petite mort» que lui impose Erich tho, enfin le cauchemar de la chute et de la dépression.

Elixir de mythes, étrange brouet de sorcière, Erichtho est donc le lieu de rencontre de figures et de thèmes mythiques d'origine culturelle diverse. Les échos mythiques qu'on y perçoit sont souvent affaiblis : Marston ne cite aucune des figures qui lui ont, à n'en pas douter, servi dans l'élaboration de ce personnage et auxquelles il fait explicitement référence dans son oeuvre antérieure (les Lamies, Héra 28 ) ; il a, en outre, gommé les références explicites aux figures de la mythologie antique (Médée, Hécate, par exemple) que contient le livre VI de La Pharsale. Mais il procède par récupération et par bricolage, ce qui est bien une des caractéristiques de la pensée mythique ${ }^{29}$.

Une autre caractéristique, généralement reconnue, de la pensée mythique est sa fonction médiatrice. Le mythe unifie les contraires en dépassant l'exclusion, d'abord posée, des deux termes : les dieux ont un aspect bénéfique et un aspect maléfique. Ils sont «bifrons» ou bien ils vont par paires. Lamia était aussi appelée Athéna. Vénus était à la fois la Céleste et la Pandémienne. Hermès est le double noir d'Apollon, Hécate le double noir d'Artémis. Une même figure est affectée de valeurs positives et de valeurs négatives. Le mythe pense fictivement la médiation, il la figure. L'élaboration de Sophonisbe et d'Erichtho relève, d'une certaine manière, de cette logique de l'ambiguité. Ainsi, Erichtho est sur toute une série de points l'antithèse de Sophonisbe. L'une est vieille et laide, l'autre est jeune et belle. L'une est animée par la luxure, l'autre est chaste. L'une est liée aux registres de la pourriture, de l'illusion et du mensonge, l'autre aspire à la pureté et à la transparence. L'une est la femmecannibale, l'autre est une denrée comestible, appétissante, que convoite en vain Syphax ${ }^{30}$. L'une est nécrophile et nécrophage, l'autre pense sans cesse à se donner la mort, mais la mort est pour elle envolée hors du monde impur. 
L'une est impie et s'efforce, par des pratiques magiques, de contraindre les puissances supérieures. L'autre est pieuse et s'incline devant ces puissances. L'une est la femme tellurique (Eri-chtho, la très terrienne ${ }^{31}$ ), un démon chthonien, l'autre est la femme-lune, froide et distante comme la chaste Phébé qu'elle invoque (III, 1, v. 122, p. 277) ; elle est, par certains aspects, une sainte ${ }^{32}$. Un contraste aussi pur, terme à terme, implique un lien de genèse réciproque et l'identité originelle de ces deux figures, qui sont inséparables l'une de l'autre ${ }^{33}$. On peut supposer que , par souci d'économie de moyens matériels, lors d'une représentation théâtrale, une seule et même personne les représentait, puisqu'elles ne sont jamais présentes ensemble sur le plateau. Erichtho/Sophonisbe, c'est le même personnage qui change de signe.

La manière dont est organisé le contenu, dans la pièce, relève elle aussi, dans une certaine mesure, de la pensée mythique. L'agencement de l'action n'est pas sans affinités avec l'agencement des récits mythiques tel que le définissent les études structuralistes. D'une part, en effet, Marston introduit dans l'action amoureuse, le motif de la quête, fondamental dans le my the ${ }^{34}$. Dans les récits de Tite-Live et d'Appien, Sophonisbe est un objet d'échange qu'Asdrubal ou les Carthaginois attribuent à Syphax ou à Massinisse selon leur intérêt ; ou bien elle s'offre elle-même à Massinisse pour échapper à la domination romaine. Jamais, dans ces récits, elle n'est l'objet d'une quête. Dans Sophonisbe en revanche, la quête amoureuse dont elle est l'objet se poursuit d'un bout à l'autre de la pièce. D'autre part, de même que dans les récits mythiques, où la quête, souvent, s'articule en trois mouvements ${ }^{35}$, l'action est soumise à une division ternaire : c'est à trois reprises que Sophonisbe subit les assauts de Syphax. Par trois fois elle se dérobe, recommençant ainsi le geste destiné à susciter l'admiration du spectateur. En outre, on s'accorde aujourd'ui à penser que le récit mythique se prête à une lecture verticale tout autant qu'horizontale, en raison de sa structure, qui est isotopique tout autant que linéaire. En se déroulant, le 
récit mythique s'enroule sur lui-même ${ }^{36}$. Or l'intention poétique avouée par Marston témoigne non seulement de sa volonté d'inventer une fiction porteuse de vérité, mais aussi d'organiser la fiction, de lui donner forme en en resserrant le sens. L'intention formalisante du poète se manifeste dans les parallélismes qu'il a établis entre diverses séquences, dans les reprises de schémas narratifs. Quant au système des personnages, il est riche en échos, en personnages-anaphores." Les mêmes valeurs, au sens musical du terme , sont reprises, légèrement augmentées ou diminuées. Le texte se cite lui-même ${ }^{37}$.

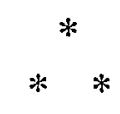

Et pourtant, la composition de la pièce dans son ensemble relève bien plutôt de la pensée rationnelle que de la pensée mythique.

Certes, pensée mythique et rationalité ne s'opposent pas radicalement. Le mythe est bien déjà une ébauche de discours rationnel. Mais alors qu'il exprime par le détour de la fiction les vérités qu'il pressent, c'est de manière directe, en empruntant la voie du raisonnement, que la philosophie formule ces vérités ${ }^{38}$. Or dans Sophonishe, les personnages sont enclins à emprunter une démarche discursive. Ils font les raisonneurs, tirent la morale des événements. Erichtho, par exemple, tire explicitement la leçon des pratiques de Syphax, ce qui donne à l'épisode l'allure d'un conte moral (V, 1, v. 23, p. 298). C'est le rationnel qui domine dans les nombreuses sentences qu'énoncent tous les personnages. Les sentences sont comme des ilôts de rationalité : en elles, l'histoire est moralisée et le mythe s'évapore.

En outre, c'est d'une logique de la contradiction, qui ne concilie pas les termes posés comme exclusifs l'un de l'autre, que relève la composition du système des personnages dans son ensemble. Deux polarités antithétiques caractérisent la configuration de l'imaginaire marstonien. Un jeu d'antinomies conceptuelles non dépassées est éta- 
bli : certains personnages ressortissent, selon la terminologie de G. Durand, au régime diurne de l'imaginaire, d'autres ressortissent au régime nocturne ${ }^{39}$. La force d'âme, la maîtrise de soi et la fidélité sont valorisées ; la mollesse sentimentale, les lâches compromissions et la gloutonnerie sont décriées. Deux mondes se font face, qui s'opposent de manière irréductible. Quant aux figures de Sophonisbe et d'Erichtho, une incertitude sémantique subsiste. Pris dans une dialectique du même et de l'autre, le spectateur doit-il percevoir Sophonisbe et Erichtho comme une figure unique affectée à la fois de valeurs positives et de valeurs négatives ? Ou s'agit-il de deux figures distinctes dont l'une serait affectée de valeurs positives et l'autre de valeurs négatives ? Autrement dit, leur élaboration relève-t-elle d'une logique de l'ambiguité ou de la contradiction ? $\mathrm{Au}$ vu du fait qu'elles prennent le relais l'une de l'autre sur le plateau, peutêtre faut-il considérer que ce rythme alternatif, cette opération de bascule occupe une position médiane entre pensée mythique et pensée rationnelle : c'est tour à tour que sont attribuées à une figure unique des valeurs contradictoires.

Enfin, à l'inverse du matériau mythique, la pièce se prête mal à l'investissement de la sensibilité. Alors que le mythe procède du mode de pensée symbolique (tout objet du matériel mythique est, dit-on, surdéterminé, polyphonique), c'est le mode de pensée allégorique qui gouverne l'inspiration de Marston. L'allégorisation n'est pas parfaite, mais le commentaire qu'appelle les personnages, même les plus complexes, ne peut aller que dans une ou quelques directions. Erichtho, par exemple, cherche à assouvir des convoitises dont l'objet est relativement vague : ce sont des rois qu'elle convoite. Elle est une abstraction morale, la luxure objectivée. Sophonisbe, quant à elle, est un modèle, «a pattern/Of what can be in a woman» dit Massinisse (I, 2, v. 228-229, p. 252). Personnage d'une pureté idéelle, elle réunit toutes les perfections que peut concevoir Massinisse. En elle, est objectivée une force morale efficace qui lui permet de tenir Syphax à distance par sa simple présence. Pure qualité devenue visible, elle in- 
carne la Vertu, charge lourde à assumer pour une princesse dans la fleur de l'âge. Les personnages n'ont pas la marge d'équivocité, le halo d'indécision qui est propre au matériau mythique. Ils incarnent, avec une netteté diagrammatique, des qualités abstraites. Or, comme Eros, les créations de l'imaginaire s'évanouissent lorsque le Poète allume la lampe de Psyché. La pièce procè de d'une élaboration intellectuelle. Son emprise sur l'imagination vient de sa construction rigoureuse, mais elle apparait souvent comme une mosaïque, un amusement de l'esprit. Un ordre formel si voyant est imposé que l'émotion reste captive dans le réseau de relations qui est établi.

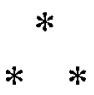

Histoire et mythe sont deux pôles qui ont orienté la création littéraire dans la Sophonisbe de Marston. Mais des contraintes autres que la référence à l'histoire antique ou à des modèles mythiques ont joué dans l'élaboration du texte. L'histoire et la mythologie ne sont ni respectées ni parodiées. La pièce n'est ni un Tite-Live travesti, ni un Ovide travesti. Elle relève d'une poétique de la rupture, qui est celle de toute une génération d'écrivains au tournant du siècle. Sans faire table rase du passé, Marston proclame bien haut le droit du Poète à inventer.

La fiction qu'il invente ressortit à la pensée rationtionnelle plus qu'à la pensée mythique. Bien que le Poète revendique la fureur sacrée de l'inspiration poétique, la pièce n'est pas un objet où le lecteur peut investir sa sensibilité. Elle est peu apte à susciter de vives réactions affectives, aujourd'hui en particulier, où l'on est peu sensible aux pompes de l'héroïsme et où les personnages de théâtre s'acheminent vers «la plus petite grandeur» 40 : la conscience morale, le mental collectif, de notre temps est mal accordé à l'éthique de la grandeur qui est valorisée dans la pièce. Mais si, comme le veut tout un courant de la critique contemporaine, l'on situe la spécificité poétique 
dans les relations entre les parties du texte (41), il est certain que le fait historique, dans la Sophonisbe de Marston, est devenu un objet, non pas mythique, mais éminemment poétique.

J. CHAUCHAIX 
NOTES

1. Voir Ch. Ricci, Sophonisbe dans la Tragédie classique italienne et française (Grenoble : Imp. Allier Frères, 1904) et A. J. Axelrad, Le Thème de Sophonisbe dans les principales tragédies de la littérature occidentale (Fran. ce, Angleterre, Allemagne), Travaux et Mémoires de 1'Université de Lille, N.S. Droit et Lettres n: 28 (1956). Cet épisode a, en particulier, fourni matière à un certain nombre d'opéras à partir du début du XVIIIe siècle (voir Ch. Ricci, op. cit., pp. 212-221 et A. J. Axelrad, op. cit., p. 182). Les documents iconographiques sont rares (voir néanmoins A. J. Axelrad, op. cit., p. 184). A noter qu'un cinéaste italien, Carmine Gallone, a repris cet épisode, de manjère rapide et sans grand succès, dans une évocation de la conquête de Carthage par les Romains (Scipion l'Africain, 1937 ; voir F. Chevassu, Scipion l'Africain, Image et Son, n? 114 (juillet 1958), pp. 43-44).

2. R. Caillois écrit, dans Le Mythe et l'homme (Paris : Gallimard, 1938), p. 31, que le mythe est «une puissance d'investissement de la sensibilité».

3. Selon R. Barthes, "Tout peutêtre mythe», Mythologies (Paris : Le Seuil, 1957), p. 216. Cf. Cl. Abastado, Mythes et rituels de l'écriture (Bruxelles: Complexe, 1979), p. 14.

4. Défaut d'inspiration mythique imputable aux auteurs ou absence de virtualités mythiques dans le personnage lui-même ? La seule manière de prouver, sans contredit, la validité de la première hypothèse et d'invalider la seconde serait d'écrire, après tant d'autres, une Sophonisbe qui attesterait l'émergence de Sophonisbe en tant que figure mythique. Disons néanmoins, sans rien prouver, que nous inclinons pour la première hypothèse, tout en reconnaissant que certains objects sont en eux-memes, et plus que d'autres, riches d'une puissance symbolique et archétypale. Ils concentrent en eux un fonds d'images plus riches et sont donc plus aptes à être entourés, par des individus ou par une collectivité humaine, d'un halo imaginaire. (Sur les rapports que l'image, le symbole et l'archétype entretiennent avec le mythe, voir G. Durand, Les Structures anthropologiques de limaginaire (Paris : Bordas, 1969 (1960), pp. 60-65).

C'est un fait notable, en tout cas, que le personnage de Sophonisbe n'a jamais, à notre connaissance, donné lieu qu'à des études de "thème» (au sens de sujet général d'une oeuvre artistique) : A. J. Axelrad intitule son étude Le Thème de Sophonisbe ; $\mathrm{R}$. Trousson, quant à lui, cite Sophonisbe à de multiples reprises dans Thèmes et Mythes (Bruxelles : Ed. de l'Université de Bruxelles, 1981), mais il choisit, dès les premières pages de son étude, de renoncer, de manière générale, à l'emploi du terme mythe, "décidément propice à toutes les confusions» (p. 20). Suivant la distinction établie par P. Albouy entre «my the» et "thème» (Mythes et mythologies dans la littérature française (Paris : A. Colin, 1969), p. 9), il faut attribuer le nom de my the aux créations proprement palingénésiques. Or, concernant Sophonisbe, les données de l'histoire antique ont été véritablement renouvelées, comme le montrent les études d'A. J. Axelrad et de R. Trousson; va-t-on, pour autant, sans hésiter, parler du «mythe de Sophonisbe»?

5. La Carthaginoise, c'est le titre choisi par Montchrestien pour la tragédie qu'il pubia vers 1596 . 
6. Il se peut que la Sophonisbe de N. de Montreux (1601) et une nouvelle de Bandello traduite par W. Painter (1566) aient incité Marston à lire les historiens antiques. Mais ces oeuvres littéraires ne peuvent pas être considérées comme des sources immédiates de la pièce. La dette de Marston envers elles consiste tout au plus dans la stimulation qu'il a reçue au départ. Voir à ce sujet J. Chauchaix, $L a$ Sophonisbe de J. Marston, Genèse et système des personnages. Essai méthodologique, Thèse dactylographiée pour le Doctorat de Troisième Cycle, présentée devant l'Université Ly on II (1983), vol. I, p. 37 et pp. 313-315.

7. Pour un inventaire des points communs aux récits des historiens antiques et à la pièce de Marston, v. ibid., pp. 41-45. Pour une analyse des différences, v. ibid., pp. $51-59$.

8. Sur l'origine des noms des personnages, v. ibid., pp. 74-93.

9. Concernant ce choix esthétique et les raisons qui peuvent l'expliquer, v. ibid., pp. 28 1-293.

i0. J. S. Colley écrit à propos de la dramaturgie marstonienne : «His is a transparent dramaturgy, a system of writing that depends upon an emphasis upon the patterns of an action rather than upon a continuous and developing narrative», John Marston's Theatrical Drama (Salzburg : s. ed., 1974), p. 45.

11. Il ne semble pas qu'en jouant avec le temps Marston cherche à produire un effet particulier. Cette prolepse narrative révèle plutôt une certaine indifférence envers la chronologie.

12. A plusieurs reprises, l'action atteint un point d'équilibre particulièrement instable où , en termes d'analyse sémiotique, la conjonction sujetobjet semble devoir se réaliser. Mais des obstacles surviennent qui empêchent la transformation de conjonction d'avoir lieu.

13. John Marston, The Wonder of Women or the Tragedy of Sophonisba, To the General Reader, p. 235. Nous nous référons, ici et ultérieurement, à l'édition de A. H. Bullen, The Works of John Marston (Londres, 1887 ; rpt. New-York : G, Olms, Anglistica et Americana (89), 1970), vol. III, pp. 231-316.

14. Sur la double structure, à la fois historique et an historique, du mythe, voir CL. Lévi-Strauss, An thropologie structurale (Paris : Hon, 1958), vol. I, p. 231 . Selon T.S. Eliot, l'impression d'immobilité et de sérénité qui est produite, dans la pièce, est semblable à celle que l'on ressent par un jour d'été ensoleillé (Selected Essay's, London : Faber and Faber , 1966), p. 232.

15. La composition de mythes est l'affaire du poè te (Platon, Phédon; Oeuvres complètes (Paris : Gallimard, 1959), tome I, p. 772). L'extase que les dieux procurent au poète lui permet de contempler les choses célestes (Ibid., Ion, pp. 62-63). Bien que $\mathrm{Pl}$ aton ait formulé diverses objections contre la poésie, son oeuvre, on le sait, contient la réponse à ces objections : n'a-t-il pas lui-même orné ses dialogues de mythes?

16. T. S. Eliot, Selected Essays, p. 229 et p. 232 .

17. M. Hours-Miédan, Carthage (Paris : P. U. F., 1959). p. 56 et pp. 
98-99.

18. Cette partition très nette entre deux mondes, thumain et le divin, n'implique pas, bien au contraire, que soit niée la dépendance de l'homme vis-à-vis de dieux lointains. Mais il est vrai que, tout au long de la pièce, l'accent est mis sur l'affirmation du moi : l'obstacle de la servitude métaphysique de 1 homme est un tremplin où rebondit l'énergie de l'individu.

19. Lucain, La Guerre civile (La Pharsale) trad. A. Bourgery (Paris : Les Belles Lettres, 1926), tome II, VI, pp. 22.42.

20. Contrairement à ce qu'écrit A. J. Axelrad dans Un Malcontent élisabéthain : John Marston (15 76-1634) (Paris : s. ed., 1955), p. 111.

21. Syphax l'invoque afin d'attirer à lui Sophonisbe qu'il désire : mais en dépit de ce que lui laisse croire la magicienne, elle ne peut pas faire naître l'amour dans les coeurs insensibles (V, 1, v. 18-20, p. 298). Dans La Pharsale, en revanche, ce pouvoir est attribué à toutes les magiciennes thessaliennes (VI, v. 452454, p. 24).

22. Apulée, Les Métamorphoses, trad. P. Vallette (Paris : Les Belles Lettres, 1940), pp. $32-93$.

23. Ibid., p. 114.

24. Voir à ce sujet A. Harris, Night's Black Agents (Manchester : Manchester Univ. Press, 1980), p. 67.

25. Voir J. Céard, «Folie et Démonologie eu XVIe siècle», Folie et Déraison à la Renaissance, Travaux de l'Institut pour l'étude de la Renaissance et de l'Humanisme, n? 5 (Bruxelles : Editions de l'Université de Bruxelles, 1976), p. 137.

26. La référence à l'enfer $(V, 1, v .23, p, 298)$ et l'introduction du thème de la chute (v. 40, p. 299) renvoient à la figure mythique du diable. Mais cette figure est aussi connotée par des indications de décor (voir les référeces au monde des ombres, de la nuit et de la mort, IV, 1, v. 94-170, pp. 290-294).

27. Voir S, Anglo, "Melancholia andWitchcraft», Folie et Déraison à la Renaissance, p. 137.

Envisagé du point de vue de l'histoire de la sensibilité, l'artifice du songe amoureux est fréquent, selon C. G. Dubois, dans le traitement maniériste de J'amour (Le Maniérisme, Paris : P.U.F., 1979, p. 200). Selon G. Mathieu-Castellani, il est caractéristique de l'érotique baroque (Mythes de l'Eros baroque, Paris : P.U.F., 1981 , pp. 135-136).

28. Marston fait référence aux Lamies dans la satire $V$ des certaine Satyres (The Poems of John Marston, A. Davenport ed. Liverpool : Liverpool Univ. Press, 1916 , v. 1, p. 87), et au mythe d'Ixion dans la satire I, v. 55 . p. 68 et dans The Metamorphosis of Higmalion's Image, strophes 16-18, pp. 55-56. Il tenait probablement une bonne part de son érudition mythologique de sources indirectes. Voir à ce sujet et, plus généralement, sur la faveur don $t$ jouissaient en Angleterre, à la fin du XVIe siècle et au début du XVIIe, les manuels de V. Cartari (Imagines Deorum) et de N. Conti (Mythologiae), J. 
Seznec, La Survivance des dieux antiques (Gap : Imp. L. Jean, 1939), pp. 279-282.

29. L'analogie qui existe entre pensée mythique et bricolage a été analysée par Cl. Lévi-Strauss, dans La Pensée sauvage (Paris : Plon, 1962), pp. 31-33.

30. Une longue tirade de Syphax sur les pratiques de la sorcière (IV, 1, v. 110-120, p. 291) plonge le lecteur dans un univers alimentaire; le motif cannibalique est souligné avec force par les références précises qui sont faites à divers processus de destruction-grattage, morsure, rongement. Quant à l'avidité sexuelle d'Erichtho $(V, 1, v .11$ et v. 15, p. 298), elle est à rapprocher de l'appétit glouton qui meut Syphax (III, 1, v. 25, p. 272 et IV, 1, v. 62 , p. 289 ).

31. Cette étymologie n'est certes pas irréfutable. Nous la proposons parce qu'aucune n'est attestée dans le dictionnaire de P. Ch antraine (Dictionnaire étymologique de la langue grecque, Paris : Klincksieck, 1968).

32. La «vertu» de Sophonisbe (IV, 1, v. 75, p. 289) est bien une force agissante aux effets surnaturels, la vertu en acte d'une sainte (voir $J$. Chauchaix, La Sophonisbe de John Marston, p. 204, no te 762 et p. 218).

33. Sur la fonction médiatrice du mythe en général, voir M. Jalley, «Le Mythe ou la raison jugée», Les Mythes, Les Cahiers de Fontenay, n:9-10 (mars 1978), pp. 240-250. Voir aussi ce qu'écrit O. Rank, dans une perspective psychanaly tique, sur la "décomposition», l'un des processus psychiques caractéristiques de l'imagination formatrice des mythes, dans Don Juan et le double (Paris : Payot, 1973), p. 252.

Pour qui voudrait sonder l'inconscient du poète, les figures et thèmes my thiques relevés précédemment (la femme objet de fascination et de terreur, mais impossible objet d'amour, la femme dévorante, le désir assouvi en rêve) semblent constituer un ensemble relativement riche et cohérent d'informations sur l'érotique marstonienne. Mais on peut préférer les rapporter à un moment spécifique de 1 histoire des modes de sensibilité : ils sont, d'après G. M. Castellani, des constantes de l'érotique baroque (Mythes de l'Eros baroque, passim). Dire que les my thes de l'érotique baroque répondent à la problématique personnelle de l'auteur exigerait une étude de l'oeuvre marstonienne dans son ensem ble.

34. Voir, par exemple, A. J. Greimas, Sémantique structurale (Paris : Larousse, 1972 (1966), pp. 180-181.

35. A. J. Greimas, Sémantique structurale, p. 4.

36. C. Lévi-Strauss compare le récit mythique à une partition d'orchestre, parce qu'il se lit diachroniquement (de gauche à droite), mais aussi synchroniquement (de bas en haut) (An thropologie structurale, vol. I, p. 234).

37. Voir J. Chauchaix, La Sophonisbe de J. Marston, pp. 278-281 et pp. $387-388$.

38. J. P. Vernant écrit, dans Mythe et société en Grèce Ancienne (Paris : Maspéro, 1974), p. 213 : «La philosophie grecque prolonge[le mythe] ou le transpose sur un autre plan en le débarrassant de ce qui en constituait 
l'élément de pure «fable». La philosophie peut donc apparaître comme une tentative pour formuler, en le démythisant, cette vérité que le mythe pressen. tait déjà à sa façon». p. 59.

39. G. Durand, Les Structures anthropologiques de l'imaginaire,

40. I. Omesco, La Métamorphose de la tragédie (Paris : P.U.F., 1978), p. 110

41. C'est sur un postulat de cohérence que s'appuie R. Jakobson ("Qu'est-ce que la poésie ?», Poétique, n:7 (1971), pp. 299-309). Ce postul at est contesté par T. Todorov (《Théories de la poésie», Poétique, n:24 (1976), p. 389 ). 